Progress in Flight Physics 5 (2013) 557-568

DOI: $10.1051 /$ eucass/201305557

(C) Owned by the authors, published by EDP Sciences, 2013

\title{
EXPERIMENTAL STUDY OF NONEQUILIBRIUM DISSOCIATION OF MOLECULAR OXYGEN
}

\author{
N. G. Bykova, L. B. Ibraguimova, O. P. Shatalov, \\ Yu. V. Tunik, and I. E. Zabelinskii
}

Institute of Mechanics

M. V. Lomonosov Moscow State University 1 Michurinsky Prosp., Moscow 119192, Russia

\begin{abstract}
The quantitative experimental data on the evolution of gas temperature $(T)$ and vibrational temperature $\left(T_{v}\right)$ of molecular oxygen behind the strong shock wave front $(T=4,000-10,800 \mathrm{~K})$ were used for determination of oxygen vibrational relaxation time and dissociation rate constants under thermal nonequilibrium conditions. Some of theoretical models of thermal nonequilibrium dissociation were tested using the measured profiles of the $T_{v}$ and $T$.
\end{abstract}

\section{INTRODUCTION}

The experimental data on the vibrational temperature $T_{v}$ of molecules during dissociation process are informative characteristics for the analysis of the relationship between the rates of vibrational relaxation and dissociation and for the development of theoretical models of these processes in thermal nonequilibrium conditions. The evident lack of such data has already resulted in a catastrophic increase in the number of models of thermal nonequilibrium dissociation [1]. The method of the vibrational temperature measurement behind the shock front under thermal and chemical nonequilibrium $\left(T_{v} \neq T\right)$ was described earlier in [2]. In the present work, the profiles of the vibrational temperature $T_{v}$ and other parameters of gas flow behind the strong shock front were used for determination of oxygen dissociation rate constants and vibrational relaxation time at temperatures up to $10,000 \mathrm{~K}$. This allows the testing of some theoretical models of thermal nonequilibrium dissociation. 


\section{RESULTS OF THE MEASUREMENTS OF THE VIBRATIONAL TEMPERATURE}

The technique of oxygen vibrational temperature measurement behind the shock front is based on the measurement of oxygen absorption from different vibrational levels and comparison of measured absorption with detailed absorption spectra calculated for vibrational $\left(T_{v}\right)$ and translational $(T)$ temperatures up to $10,000 \mathrm{~K}$. This technique was described earlier in [2] and was employed using several wavelengths from 200 to $260 \mathrm{~nm}$. The test gas was undiluted high purity oxygen (99.999\%, Linde Production) with the initial pressures ranging from 1 to 2 Torr. The shock velocity was changed from 3 to $4.5 \mathrm{~km} / \mathrm{s}$. The temperatures $T_{0}$ directly within the shock front were from 4,000 to $10,800 \mathrm{~K}$. The spectrograph selected a narrow spectral region, while the width of the instrument function was $1 \mathrm{~nm}$. This made it possible to attribute the measurements separated by an interval of 10 to $30 \mathrm{~nm}$ to absorption from different vibrational levels of oxygen molecules.

The example of the vibrational temperature profile behind the shock front is presented in Fig. 1. The rapid growth of the temperature $T_{v}$ falls for a time of the order of 1 to $2 \mu \mathrm{s}$ in all the regimes considered. The chemical (thermodynamic) equilibrium is attained at a time greater than $10 \mu \mathrm{s}$. In Fig. 1, the experimental points were obtained using absorption oscillograms corresponding to three different wavelengths $(220,230$ and $260 \mathrm{~nm})$. The uncertainty of temperature determination was not higher than $10 \%[2]$.

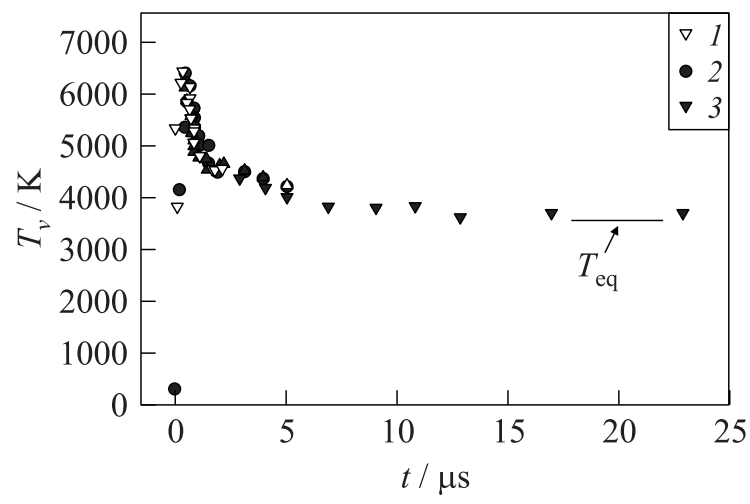

Figure 1 The profile of vibrational temperature $T_{v}$ behind the front of shock wave in oxygen at $T_{0}=8620 \mathrm{~K}$ (temperature directly in front). The symbols are the measured values $T_{v}$ using absorption oscillograms at wavelengths 220 (1), 230 (2), and $260 \mathrm{~nm}(3)$; $T_{\text {eq }}$ is the equilibrium temperature 


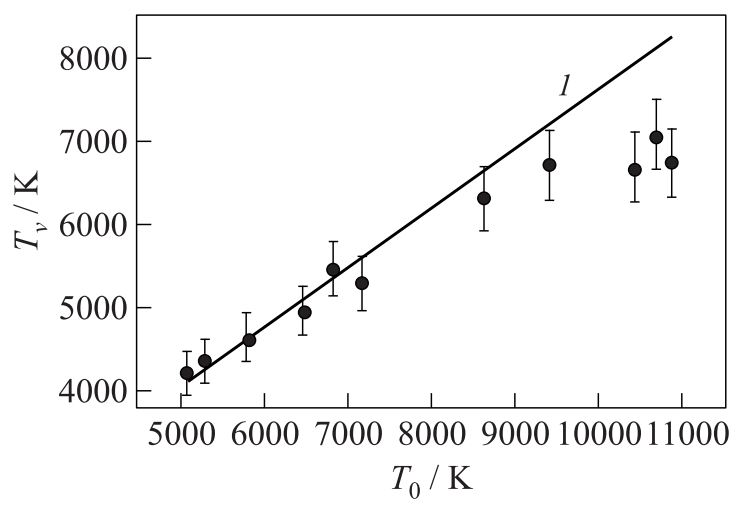

Figure 2 Maximum vibrational temperature $T_{v}^{\max }$ behind the shock front vs. $T_{0}: 1-$ temperature $T_{1}$ calculated under assumption of vibrational-translational equilibrium before the dissociation onset. Signs refer to experimental data

At temperatures $T_{0}<6500 \mathrm{~K}$, the maximum vibrational temperature $T_{v}^{\max }$ was attained for a time from 0.5 to $1.5 \mu \mathrm{s}$, while for the higher values of $T_{0}$, this time shortened to $0.2-0.4 \mu \mathrm{s}$ (see Fig. 1). At these times, the temporal resolution $\Delta t \sim 0.1 \mu$ s was attained $(\Delta t=\Delta s / V$ where $\Delta s=0.3 \mathrm{~mm}$ is the width of optical slit and $V$ is the shock front velocity). This allowed to process the absorption curves with the step $\Delta t$, with confidence that the absorption measured at a certain time was not averaged by absorption from the neighboring regions of shock wave.

With increase in the temperature $T_{0}$ within the shock front, the vibrational temperature profiles modified, namely, the maximum value was attained faster and the difference between $T_{0}$ and the temperature in the equilibrium region grew. On the oscillograms, the temporal interval of the kinetic region behind the shock front shortens. Earlier, it was revealed [2] that in the temperature range $T_{0}=3500-6500 \mathrm{~K}$, there is agreement between the temperature $T_{1}$ calculated under the assumption of vibrational-translational equilibrium before the dissociation onset and the temperature $T_{v}^{\max }$ measured at the absorption maximum (Fig. 2). This result confirms the well-known assumption of the separation of the vibrational relaxation and dissociation zones on the abovementioned temperature range. At the same time, it can be seen that with increase in the temperature $T_{0}$, the difference between the maximum vibrational temperature $T_{v}^{\max }$ and the temperature $T_{1}$ progressively grows. Already for $T_{0} \sim 6500 \mathrm{~K}$, the measured value of $T_{v}^{\max }$ is lower from 100 to $200 \mathrm{~K}$ than the calculated values of $T_{1}$. With further increase in $T_{0}$, the difference between $T_{1}$ and the measured value of $T_{v}^{\max }$ continues to grow and reaches $1500 \mathrm{~K}$ at $T_{0} \sim 10,500 \mathrm{~K}$. 


\section{DETERMINATION OF THE GAS FLOW PARAMETERS BEHIND THE SHOCK FRONT}

Behind the front of a stationary shock wave, the gas flow is governed by the equations of conservation of the fluxes of mass, momentum, energy, and component composition:

$$
\left.\begin{array}{c}
\rho_{1} V=\rho_{2} v_{2} ; \quad p_{1}+\rho_{1} V^{2}=p_{2}+\rho_{2} v_{2}^{2} ; \\
p=\rho R T \sum_{i} \gamma_{i} ; \quad \frac{1}{\mu}=\sum_{i} \gamma_{i} ; \\
\sum_{i} \gamma_{i}^{(2)} H_{i}^{(2)}+\frac{v_{2}^{2}}{2}=\sum_{i} \gamma_{i}^{(1)} H_{i}^{(1)}+\frac{V^{2}}{2} ; \\
2 \gamma_{1}^{(1)}=0.0625=2 \gamma_{1}^{(2)}+\gamma_{2}^{(2)} .
\end{array}\right\}
$$

Here, $V$ is the shock velocity; $v_{2}$ is the gas flow velocity relative to the shock front; $T, p$, and $\rho$ are the gas temperature, pressure, and density; $\mu$ is the molecular weight of the gas mixture; and $H$ is the enthalpy of oxygen molecules and atoms. The state of the gas is denoted by the superscripts and subscripts $(1-$ ahead of the shock front and 2 - behind it). The molar-mass concentration $\gamma_{i}$ is presented in the form:

$$
\gamma_{i}=\frac{n_{i}}{\rho N_{A}} .
$$

Here, $n_{i}$ is the oxygen atoms or molecules concentration and $N_{A}=6.02$ $\times 10^{23} \mathrm{~mole}^{-1}$ is the Avogadro number. The enthalpy $H_{i}(T)$ for oxygen atoms and molecules is taken from [3]. The part of the enthalpy corresponding to the vibrational energy of oxygen molecules was presented in the form $\varepsilon(T)$ $=H_{\mathrm{O}_{2}}(T)-(7 / 2) R T$. The values of $H_{\mathrm{O}_{2}}(T)$ and $\varepsilon(T)$ were approximated by third-power polynomials.

In the solution of system (1), the parameters of gas flow behind the shock front such as temperature, pressure, density, and molar-mass concentrations of oxygen molecules and atoms were obtained for each time using the measured values of vibrational temperature, concentration of molecules, and initial conditions ahead of shock front. The oxygen molecule concentration $n_{\mathrm{O}_{2}}^{(2)}$ was determined from the absorption oscillograms using Beer law and the measured vibrational temperature $T_{v}$ :

$$
n_{\mathrm{O}_{2}}^{(2)}=-\frac{1}{\sigma l} \ln \frac{I}{I_{0}}
$$

where $I / I_{0}$ is the measured fraction of the passing radiation; $\sigma=\sigma\left(T, T_{v}\right)$ is the spectral absorption cross section per molecule (in $\mathrm{cm}^{2}$ ); $l$ is the optical path length in the absorbing gas (the inside diameter of the shock tube in $\mathrm{cm}$ ). For the 


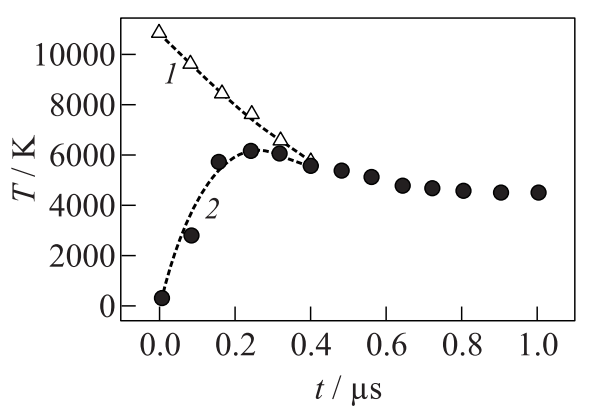

(a)

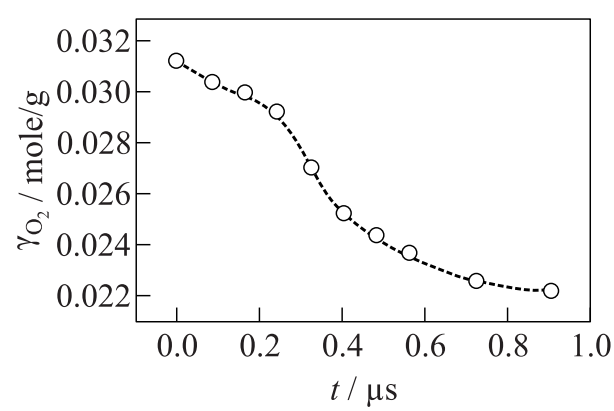

(b)

Figure 3 Profiles of the translational $T_{2}(1)$ and vibrational $T_{v}(2)$ temperatures $(a)$ and the molar-mass oxygen concentration $\gamma_{\mathrm{O}_{2}}^{(2)}$ behind the shock front $(b)$ at $p_{1}=0.8$ Torr and $V=4.44 \mathrm{~km} / \mathrm{s}\left(T_{0}=10,820 \mathrm{~K}\right)^{2}$

wavelengths used in the experiments, the absorption cross sections $\sigma=\sigma\left(T, T_{v}\right)$ are known [2].

In the process of solution, a steep dependence of the determined quantities $T_{2}, \rho_{2}, p_{2}, v_{2}, \gamma_{\mathrm{O}_{2}}^{(2)}$, and $\gamma_{\mathrm{O}}^{(2)}$ from the input data $n_{\mathrm{O}_{2}}^{(2)}\left(t_{\mathrm{lab}}\right)$ was revealed. The accuracy of determining the concentration needed for these calculations (tenths of one percent) cannot be provided in the experiments. For this reason, for any chosen moment of time, the calculations were performed for several values of concentration $n_{\mathrm{O}_{2}}^{(2)}\left(t_{\text {lab }}\right)$ varied within fairly narrow limits and then an analysis of the solutions thus obtained was made. As a result, the gas flow parameters were determined for each time. In Fig. 3 and in the following figures, time is given in laboratory coordinates system. After $0.4 \mu \mathrm{s}$, the vibrational temperature reaches the translational temperature $T_{2}$.

\section{OXYGEN DISSOCIATION RATE CONSTANTS IN THERMALLY NONEQUILIBRIUM CONDITIONS}

The calculated profiles of the parameters $T_{2}(t), n_{\mathrm{O}_{2}}^{(2)}(t), \gamma_{\mathrm{O}_{2}}^{(2)}(t)$, and $\rho_{2}(t)$ make it possible to determine the $\mathrm{O}_{2}$ molecule dissociation rate constants $k_{d}$ both under thermal equilibrium $\left(T_{v}=T_{2}\right)$ and thermal nonequilibrium conditions $\left(T_{v} \neq T_{2}\right.$, near the shock front). For the molar-mass concentrations defined by Eq. (2), the kinetic equation can be presented in the form:

$$
\frac{d \gamma_{\mathrm{O}_{2}}}{d t}=k_{d} \gamma_{\mathrm{O}_{2}}^{2} \rho_{2}
$$




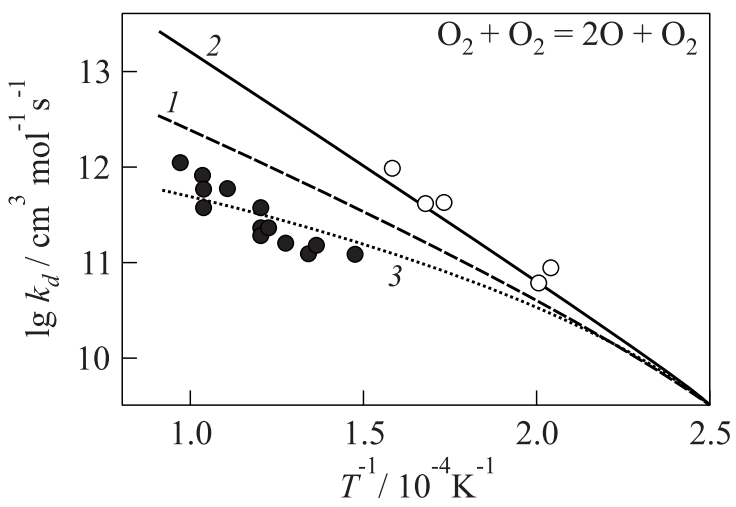

Figure 4 Dissociation rate constant $k_{d}\left(\mathrm{O}_{2}-\mathrm{O}_{2}\right)$ vs. $T^{-1}$; filled signs refer to $T_{v} \neq T_{2}$ and empty signs to $T_{v}=T_{2}: 1-$ data [4];2- $k_{d}$ recommended in [5] for conditions $T_{v}=T ;$ and 3 - modeling

Since in determining the rate constant $k_{d}$ the processes are considered in the vicinity of the shock front, the recombination term is not taken into account. For any regime, the $\mathrm{O}_{2}$ molecule dissociation degree $\alpha$ was defined by the relation $\alpha=\left(\gamma_{\mathrm{O}_{2}}^{(0)}-\gamma_{\mathrm{O}_{2}}^{(2)}\right) / \gamma_{\mathrm{O}_{2}}^{(0)}$ where $\gamma_{\mathrm{O}_{2}}^{(0)}=0.03125$ is the molar-mass concentration of molecules at the initial moment of time behind the shock front. In processing the experimental data, it was assumed that at oxygen dissociation degrees smaller than $3 \%-5 \%$, the dissociation is determined only by the $\mathrm{O}_{2}-\mathrm{O}_{2}$ collisions, that is, the role of the newborn atoms was neglected.

The dissociation rate constants $k_{d}\left(\mathrm{O}_{2}-\mathrm{O}_{2}\right)$ thus determined are presented in Fig. 4 for the cases in which $T_{v} \neq T_{2}$ (filled signs) and $T_{v}=T_{2}$ (empty signs). The nonequilibrium dissociation rate constants $\left(T_{v} \neq T_{2}\right)$ were obtained for several high-temperature regimes $T_{0}=10,820,10,400,9,400$, and $8,620 \mathrm{~K}$. Uncertainty of rate constant was evaluated using scatter of measured data: $\Delta \lg k_{d}= \pm 0.15$. As can be seen from the figure, the values of thermal nonequilibrium dissociation rate constants $k_{d}$ determined in this study are less than data of [4] (curve 1) and much less than [5] (curve 2). The equilibrium values $k_{d}$ obtained for the regimes with $T_{0}=9,400,8,620$, and $6,470 \mathrm{~K}$ coincide with curve 2 corresponding to the equilibrium values of $k_{d}^{0}\left(T=T_{v}\right)$ recommended in [5]. For dissociation degrees $\alpha>5 \%$, the processing of experimental data gives at the same temperature the bigger values of $k_{d}$ than at $\alpha<5 \%$ owing to accelerating influence of oxygen atoms on the dissociation process. In Fig. 5, the experimental values of dissociation degree of $\mathrm{O}_{2}$ behind the shock front are presented for five regimes with different shock wave velocities. 


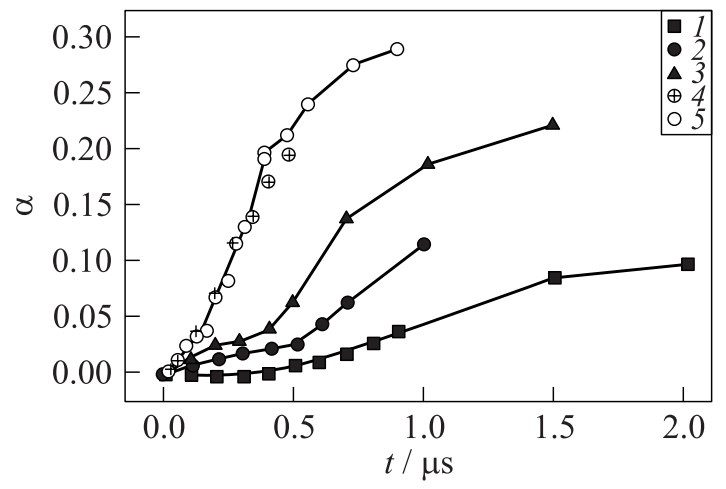

Figure 5 Experimental values of dissociation degree of $\mathrm{O}_{2}$ behind the shock front for different shock wave velocities (or $T_{0}$ ): $1-T_{0}=6,470 \mathrm{~K} ; 2-8,620 ; 3-9,410$; $4-10,400 ;$ and $5-T_{0}=10,820 \mathrm{~K}$

\section{TESTING OF THEORETICAL MODELS OF MOLECULE DISSOCIATION}

In this study, the calculations of gas flow parameters behind the shock wave front were fulfilled using the one-dimensional gasdynamics equations for a steady-gas flow by the Gear method jointly with the system of ordinary differential equations describing oxygen dissociation and accounting for the excitation of vibrational degrees of freedom. Viscosity, heat conduction, and diffusion were not taken into account. In describing the kinetics of physical and chemical processes in the gas, the classical coupled vibration-dissociation-vibration (CVDV) model [6] was used. This model allows the simultaneous dissociation and vibrational kinetics. In [6], it was assumed that the dissociation rate constant depends on the gas temperature $T$ and on the vibrational temperature of the dissociating molecule $T_{v}$ and at the same time, the mean vibrational energy of the molecule $\varepsilon$ is determined by the variation in the particle concentration as the chemical reaction proceeds:

$$
\begin{aligned}
\frac{d N_{A B}}{d t} & =-\sum_{i} k_{d i} N_{i} N_{A B}+\sum_{i} k_{r i} N_{i} N_{A} N_{B} ; \\
\frac{d \varepsilon}{d t} & =\frac{\varepsilon_{0}-\varepsilon}{\tau}-(E-\varepsilon) \sum_{i} k_{d i} N_{i}+(G-\varepsilon) \sum_{i} k_{r i} N_{i} \frac{N_{A} N_{B}}{N_{A B}} .
\end{aligned}
$$

Here, $N_{A B}, N_{A}, N_{B}$, and $N_{i}$ are the concentrations of particles $A B, A, B$, and $i$ per unit volume; $\varepsilon_{0}$ and $\varepsilon$ are the equilibrium and current vibrational energies per one $A B$ molecule; $\tau$ is the vibrational relaxation time; $k_{d i}$ and $k_{r i}$ are the 
rate constants for the dissociation and recombination of $A B$ molecules when interacting with particle $i$, respectively; and $E$ and $G$ are the mean vibrational energies lost in a single dissociation act of an $A B$ molecule or acquired upon atom recombination $A+B$, respectively. It is assumed that $E=G$. In [1], certain models of diatomic molecule dissociation under thermally nonequilibrium conditions are described and corresponding expressions for the molecule dissociation rate constants are presented. In the two-temperature representation, these models usually have the form:

$$
k_{d}\left(T, T_{v}\right)=k_{0}(T) Z\left(T, T_{v}\right) .
$$

Here, $k_{0}(T)$ is the equilibrium dissociation rate constant corresponding to the condition $T=T_{v}$ and $Z\left(T, T_{v}\right)$ is the coupling factor characterizing the influence on this constant of deviation from equilibrium of vibrational degrees of freedom. The mean vibrational energy $E$ lost in a dissociation act (Eqs. (3) and (4)) is represented by different functions in different dissociation models. For example, in the Kuznetsov model [7] tested in this work the energy $E$ is presented as a function of the vibrational temperature, while in the Macheret-Friedman model [8], it depends on the translational $T$ and vibrational $T_{v}$ temperatures. In the calculations, the oxygen dissociation rate constant from work [4] was taken as $k_{0}$, where it is presented in the form:

$$
k=A T^{-2.5} \exp \left(-\frac{59,380}{T}\right) \mathrm{cm}^{3} \mathrm{~mole}^{-1} \mathrm{~s}^{-1} .
$$

For colliding partner $M=\mathrm{O}_{2}$, the multiplier $A=9.8 \cdot 10^{24}$, while for $M=\mathrm{O}$, $A=3.5 \cdot 10^{25}$. The initial expression of vibrational relaxation time $p \tau_{\mathrm{O}_{2}-\mathrm{O}_{2}}$ was taken from [9]. In calculations, the values of $p \tau_{\mathrm{O}_{2}-\mathrm{O}_{2}}$ were varied to obtain the better fitting of vibrational temperature to the measured ones at initial steps. The values of $p \tau_{\mathrm{O}_{2}-\mathrm{O}}$ were taken from [10]:

$$
p \tau_{\mathrm{O}_{2}-\mathrm{O}}=1.5 \cdot 10^{-12} T^{0.5}\left(1-\exp \left(-\frac{2238}{T}\right)\right)^{-1} \exp \left(86.4 T^{-1 / 3}\right)[\mathrm{atm} \cdot \mathrm{s}] .
$$

For any of the models chosen for testing, the coupling factor $Z\left(T, T_{v}\right)$ depends on the assumptions and restrictions adopted by their authors. Thus, the Kuznetsov model is a model of anharmonic oscillator dissociation with account for the effective energy boundary of the rapid and slow exchange by vibrational quanta. It is assumed that dissociation proceeds mainly from the upper vibrational levels. The Macheret-Friedman model allows two dissociation mechanisms, namely, dissociation from the upper vibrational levels for vibrationally-excited molecules and from the lower levels for unexcited or weakly excited molecules. The models are described in details in $[1,8]$.

The comparison of the measured and calculated profiles of the translational and vibrational temperatures shows different patterns for the temperatures $T_{0}$ 


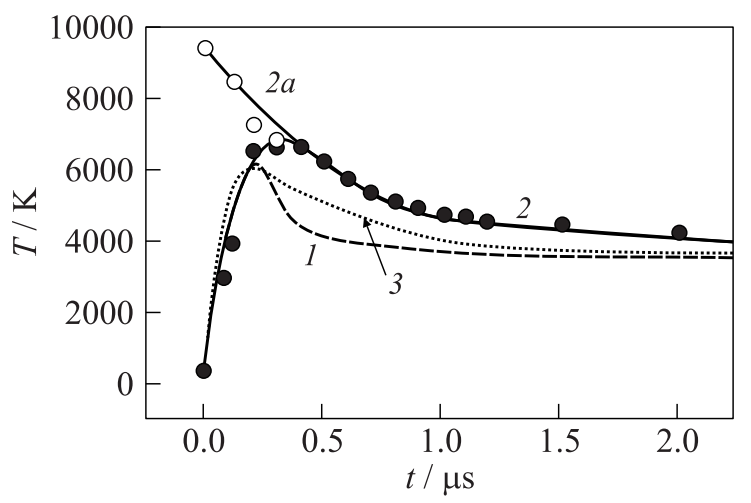

Figure 6 Comparison of the measured translational $T_{2}$ (filled signs) and vibrational $T_{v}$ (empty signs) temperatures and calculated (initial temperature $T_{0}=9,410 \mathrm{~K}$ ): 1 $T_{v}$ calculated using dissociation rate constant $k_{d}$ from Kuznetsov model; 2 and $2 \mathrm{a}-$ $T_{v}$ and $T_{2}$ calculated using Kuznetsov model with $k_{d} / 5$; and $3-T_{v}$ calculated using Macheret-Friedman model

$\leq 6,500 \mathrm{~K}$ and $T_{0}>7,000 \mathrm{~K}$. At $T_{0}<6,500 \mathrm{~K}$, the calculations with Kuznetsov model satisfactorily describe the measured $T_{v}$ profiles. However, with increase in the temperature, the same model no longer gives a satisfactory description of vibrational temperature profile (Fig. 6 , curve $1, T_{0}=9,410 \mathrm{~K}$ ). The agreement between the measured and calculated data can be achieved only by reducing the rate constants $k_{d}$ by a factor of five (curve 2). Utilization of MacheretFridman model leads to analogous result. Thus, neither of the tested models satisfactorily describes the vibrational and translational temperature profiles for $T>7,000 \mathrm{~K}$. Clearly, the models overestimate the role of dissociation in the thermal nonequilibrium zone, and the calculated degree of oxygen dissociation increases faster than it follows from the experiments.

\section{VIBRATIONAL RELAXATION OF $\mathrm{O}_{2}$ MOLECULES}

All the data on the vibrational relaxation times of molecules collected in [9] pertain to the low-temperature region $T \leq 2 \theta(\theta$ is the characteristic vibrational temperature of molecules), except for the relaxation times of oxygen molecules measured up to the temperatures of $10,200 \mathrm{~K}$ [11]. For gas temperatures lower than $2 \theta$ or slightly exceeding this value, the relaxation of vibrational energy is well described by the systematics [9] and by the adiabatic Landau-Teller theory [12]. At higher temperatures for many molecules, including oxygen molecules, the function $\lg (p \tau)=f\left(T^{-1 / 3}\right)$ deviates from the linear dependence. However, 
theory [12] takes into account some other temperature-dependent factors. According to [12], for diatomic molecules, the vibrational relaxation time is determined by the expression

$$
p \tau=\frac{k T}{\sigma_{0} \sqrt{8 R T /(\pi \mu)} p \mathrm{P}_{10}(1-\exp (-\theta / T))}
$$

where $\sigma_{0}$ is the gaskinetic effective cross section of molecule collisions with particles of the medium; $\mathrm{P}_{10} \sim \exp \left(T^{1 / 3}\right)$ is the probability of molecule deactivation from the first vibrational level; and $\mu$ is the reduced molecular weight of the colliding particles.

By means of the comparison with experimental data, expression (5) was reduced in $[10]$ to the form:

$$
p \tau=\frac{f T^{0.5}}{1-\exp (-\theta / T)}[\mathrm{atm} \cdot \mathrm{s}] ;
$$

for oxygen, $f=8.8 \cdot 10^{-14} \exp \left(172.7 T^{-1 / 3}\right)$.

At low temperatures $(T<2 \theta)$, the vibrational relaxation time depends primarily on $\mathrm{P}_{10}$, and the temperature dependence of the $\lg (p \tau)$ as a function of $T^{-1 / 3}$ is almost linear, which was shown in [9]. However, with increase in the temperature, the $T^{-1 / 3}$-dependence of $\lg (p \tau)$ deviates from the linear dependence due to the growing influence of other temperature factors in expression (6) as well as to the effect of some other factors which are not accounted for in the Landau-Teller theory, such as anharmonicity, special features of the interaction potential, etc. Figure 7 presents the vibrational relaxation times for oxygen

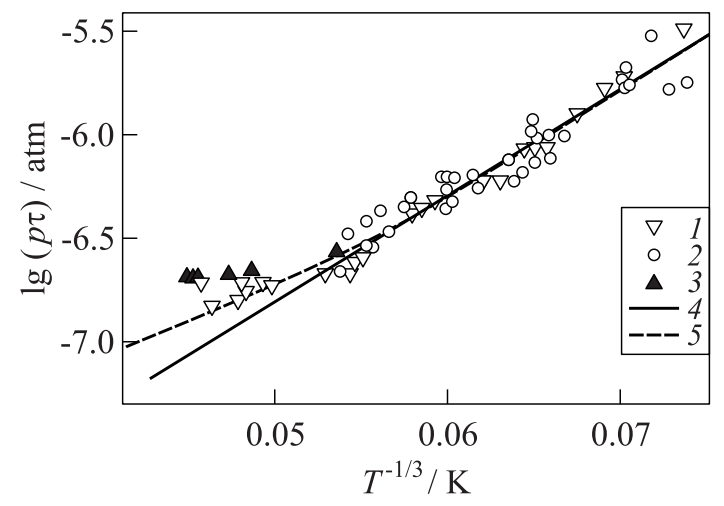

Figure 7 Vibrational relaxation time $\tau\left(\mathrm{O}_{2}-\mathrm{O}_{2}\right)$ vs. temperature $\left(T^{-1 / 3}\right): 1-$ experiments [11]; 2 - experiments [13]; 3 - data of the present work; 4 - data of [9]; and 5 - data of $[10]$ 
molecules in the $\mathrm{O}_{2}-\mathrm{O}_{2}$ medium. The data taken from [9] are shown by curve 4, while curve 5 presents the $p \tau$ values given by expression (6). The experimental points from studies $[11,13]$ are also presented. The $p \tau$ values measured in this study are shown by 3 . These values are obtained by fitting the calculated profiles of the vibrational temperature of $\mathrm{O}_{2}$ molecules behind the shock front to the experimental ones close to the shock front. Clearly, for $T_{0}<6,000 \mathrm{~K}$, the measured $T_{v}$ profiles are well described by expression given by Millikan and White in [9]. However, with increase in temperature, the values of $\tau$ must be increased in comparison with $[9,10]$. Note that under temperatures $6,000-10,000 \mathrm{~K}$, the experimental values of $p \tau$ weakly depend on temperature.

\section{CONCLUDING REMARKS}

The measurements of the profiles of vibrational and translational temperatures behind the front of a shock wave, carried out in the present work, made it possible to ascertain that the vibrational relaxation and dissociation zones are separated at $T<6,500 \mathrm{~K}$, and the vibrational-translational equilibrium is attained before the dissociation onset. At $T>6,500 \mathrm{~K}$ the vibrational relaxation of molecules proceeds already close to the shock front jointly with the dissociation, and the vibrational-translational equilibrium has no time to be attained before the dissociation onset.

The rate constants of oxygen molecule dissociation are determined for the $\mathrm{O}_{2}-\mathrm{O}_{2}$ collisions under both thermal equilibrium and thermal nonequilibrium conditions on the temperature range from 6,500 to $10,800 \mathrm{~K}$. It is shown that the models $[7,8]$ completely describe the measured temperature profiles at separated vibrational relaxation and dissociation processes (at temperatures in shock front less $6,500 \mathrm{~K}$ ). However, at the temperatures higher than 7,000 K, neither of the tested models describes the measured temperature profiles. Each of the models overestimates the role of dissociation in the thermally nonequilibrium zone.

It is shown that at $T>5,000 \mathrm{~K}$, the vibrational relaxation time of oxygen molecules decelerates by comparison with [9] and [12] dependences.

\section{ACKNOWLEDGMENTS}

The authors are grateful to Prof. A. I. Osipov, Prof. A. V. Uvarov, and Dr. E. G. Kolesnichenko for useful and fruitful discussions. We are also grateful to Dr. Alla Sergievskaya for her invaluable assistance in carrying out this study.

This work was supported by Grant RFBR No. 10-01-00327a. 


\section{REFERENCES}

1. Kovach, E. A., S.A. Losev, and A. L. Sergievskaya. 1995. Models of twotemperature chemical kinetics for description of molecule dissociation in strong shock waves. Chem. Phys. Rep. 14:1353-87.

2. Zabelinskii, I. E., L. B. Ibraguimova, O. P. Shatalov, and Yu. V. Tunik. 2012. Experimental study and numerical modeling of vibrational oxygen temperature profiles behind a strong shock wave front. In: Progress in flight physics. Eds. Ph. Reijasse, D. D. Knight, M. Ivanov, and I. Lipatov. EUCASS advances in aerospace sciences book ser. Moscow: TORUS PRESS. 3:231-42.

3. Glushko, V.P., ed. 1978. Thermodynamic properties of individual substances. Reference book. Moscow: Nauka. Vol. 1. Bd. 2. 327 p. [In Russian.]

4. Baulch, D. L., D. D. Drysdale, J. Duxbury, and S. J. Grant. 1976. Evaluated kinetic data for high temperature reactions. London: Butterworths. Vol. 3. 593 p.

5. Ibraguimova, L. B., G.D. Smekhov, and O.P. Shatalov. 1999. Dissociation rate constants of diatomic molecules under thermal equilibrium conditions. Fluid Dyn. 34:153-57.

6. Treanor, C. E., and P. V. Marrone 1962. Effect of dissociation on the rate of vibrational relaxation. Phys. Fluids 5:1022-26.

7. Kuznetsov, N. M. 1971. Kinetics of molecule dissociation in molecular gases. Theor. Exp. Chem. 7:22-33. [In Russian.]

8. Sergievskaya, A. L., S. A. Losev, S. Macheret, and A. Fridman. 1997. Selection of two-temperature chemical reaction models for nonequilibrium flows. AIAA Paper No. 1997-2580.

9. Millikan, R.C., and D.R. White. 1963. Systematics of vibrational relaxation. J. Chem. Phys. 39:3209-13.

10. Ibraguimova, L. B., G. D. Smekhov, and O.P. Shatalov. 2004. On the correct presentation of vibrational relaxation time of diatomic molecules at high temperatures. In: Physics of extreme states of matter. Chernogolovka. 97-98. [In Russian.]

11. Losev, S. A., and N.A. Generalov. 1962. On study of excitation of vibrations and decay of oxygen molecules at high temperatures. Sov. Phys. - Dokl. 6:1081-85.

12. Landau, L., and E. Teller. 1936. Theory of sound dispersion. Phys. Zs. Sow. 10:3443.

13. Bykova, N. G., I. E. Zabelinskii, L. B. Ibraguimova, et al. 2004. Numerical and experimental study of kinetic processes in atmospheric plasma. Moscow: Institute of Mechanics of Moscow State University. Report No. 4736. 66 p. [In Russian.] 